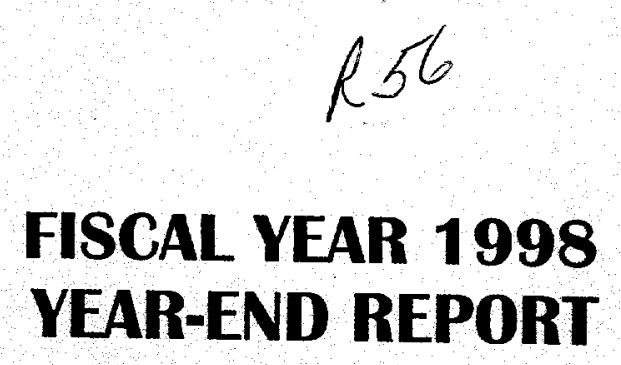

For the November 1997 to October 1998 Period

\title{
ONLINE MEASUREMENT OF THE PROGRESS OF DECONTAMINATION
}

\author{
Principal Investigator: \\ M.A. Ebadian, Ph.D. \\ Florida International University \\ Collaborators: \\ Leonel E. Lagos \\ Suresh Chegireday. \\ Sergio Wong
}

\section{Prepared for: \\ U.S. Department of Energy Office of Environmental Management Office of Science and Technology}

Hemispheric Center for Environmenin Iechnolocy (HCET) Florida International University, Center for Engineering \& Applied Sciences 10555 West Flagler Street, EAS-2100, Miami, Florida 33174 305-348-4238 - FAX: (305) 348-1852 - World Wide Web Site: http://www.hcet.fiu.edu

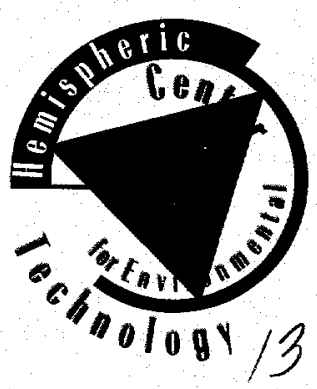




\section{DISCLAIMER}

This report was prepared as an account of work sponsored by an agency of the United States government. Neither the United States government nor any agency thereof, nor any of their employees, nor any of its contractors, subcontractors, nor their employees makes any warranty, express or implied, or assumes any legal liability or responsibility for the accuracy, completeness, or usefulness of any information, apparatus, product, or process disclosed, or represents that its use would not infringe upon privately owned rights. Reference herein to any specific commercial product, process, or service by trade name, trademark, manufacturer, or otherwise does not necessarily constitute or imply its endorsement, recommendations, or favoring by the United States government or any agency thereof. The views and opinions of authors expressed herein do not necessarily state or reflect those of the United States government or any agency thereof. 


\section{DISCLAIMER}

Portions of this document may be illegible in electronic image products. Images are produced from the best available original document. 


\title{
ONLINE MEASUREMENT OF THE PROGRESS OF DECONTAMINATION
}

\section{Principal Investigator}

M.A. Ebadian, Ph.D.

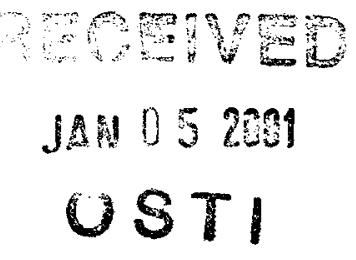

Hemispheric Center for Environmental Technology

Florida International University

Miami, FL 33174

\section{Florida International University \\ Collaborators}

\author{
Leonel E. Lagos, Suresh Chegireddy, and Sergio Wong \\ Hemispheric Center for Environmental Technology \\ Florida International University \\ Miami, FL 33174
}

January 1999

\section{Prepared for}

U.S. Department of Energy

Office of Environmental Management

Office of Science and Technology

U.S. Department of Energy

Under Grant No.: DE-FG21-95EW55094 


\section{ACKNOWLEDGMENTS}

This report is based on work supported by the U.S. Department of Energy, Office of Environmental Management, Office of Science and Technology's Deactivation and Decommissioning Focus Area, Federal Energy Technology Center. The Principal Investigator, FIU Collaborators, and students at Florida International University would like to thank Dr. Paul Hart for providing us the opportunity to work on this project. 



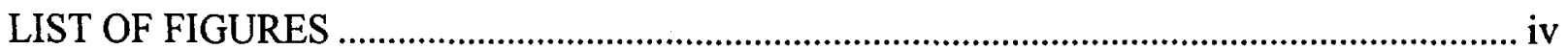

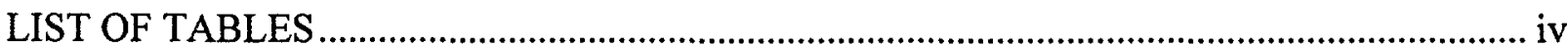

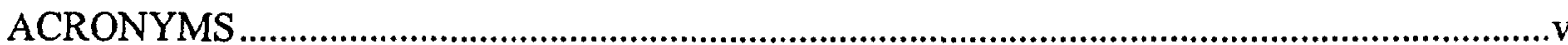

NOMENCLATURE ……...................................................................................................

EXECUTIVE SUMMARY ............................................................................................ vi

1.0 INTRODUCTION

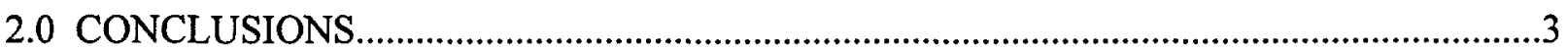

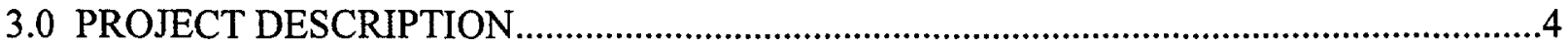

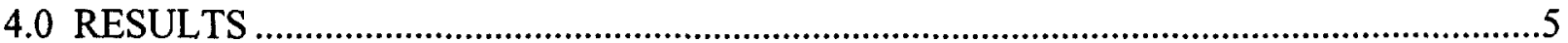

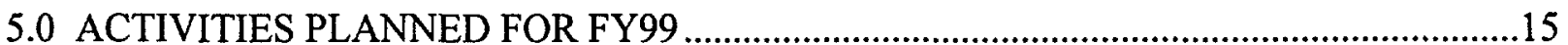

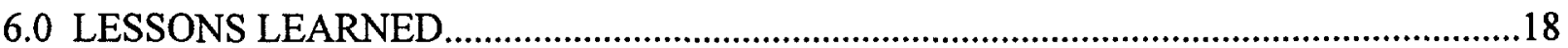

APPENDIX A. Online Measurement of the Progress of Decontamination: Requirements Document

APPENDIX B. Preliminary Feasibility Study for the Integration of a Characterization Technology with a Decontamination Technology

APPENDIX C. Empirical Feasibility Study of the Effects of Horizontal Motion on the Efficiency of Radiation Sensors 


\section{LIST OF FIGURES}

Figure 1. European Blasting Equipment 350 Centrifugal shot-blasting unit............................. 9

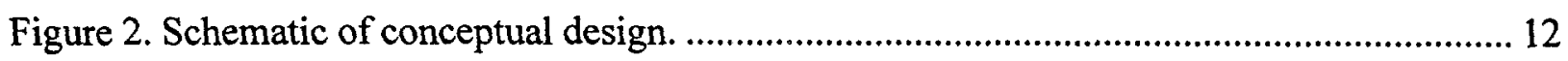

Figure 3. Conceptual drawing of integrated system. ............................................................ 13

\section{LIST OF TABLES}

Table 1. Decontamination Technologies Evaluated ........................................................... 6 


\section{ACRONYMS}

D\&D Deactivation and Decommissioning

DOE U.S. Department of Energy

DOE-EM U.S. Department of Energy-Environmental Management

DOE-OST U.S. Department of Energy-Office of Science and Technology

FIU Florida International University

FIU-HCET Florida International University-Hemispheric Center for Environmental Technology

FY97 Fiscal year 1997

FY98 Fiscal year 1998

HCET Hemispheric Center for Environmental Technology

INEEL Idaho National Engineering and Environmental Laboratory

IUOE International Union of Operating Engineers

MODM Multi-objective decision making

ORNL Oak Ridge National Laboratory

PC Personal computer

RAPIC Remedial Action Program Information Center

NOMENCLATURE

$\begin{array}{ll}\mathrm{psi} & \text { pound per square inch } \\ \mathrm{ft} & \text { foot } \\ \mathrm{V} & \text { volt } \\ \mathrm{amp} & \text { ampere }\end{array}$


The aim of this project was to develop, build, test, and possibly commercialize a decontamination system that, in addition to its regular functions, has the ability to assess the progress of decontamination by integrating real-time radiation sensors. This goal has several positive effects, such as an increase in efficiency during decontamination operations, lowering the number of radioactivity surveys that need to be performed, reducing the amount of waste produced and lowering the risk to the D\&D worker, thus lowering the cost of the overall operation. During the first year of this three-year project, the following tasks have been accomplished:

- Delineate the requirements for the final integrated unit.

- Evaluate decontamination technologies and generate a short list of technologies suitable for sensor integration.

- Investigate commercially available radiation sensors and select a subset of sensors recommended for integration.

- Technical review of the decontamination technology short list and selection of a final technology suitable for integration.

- First-round design of sensor array and integration.

- First-round design of data collection, storage and transmission system.

- First-round integration design.

The tasks for project years two and three will involve redesigns for deficiencies found during the first year of work, bench testing of prototype designs, further engineering designs and modifications, system integration testing, extensive vendor interface, delineation of integrated system performance parameters, field testing at a DOE facility, and commercialization efforts. 


\subsection{INTRODUCTION}

The Department of Energy (DOE) has estimated that 23 million cubic meters of concrete will require disposition as 1200 buildings undergo the $D \& D$ process. All concrete removal that will be performed will also necessitate extensive use of characterization techniques. As previously detailed, of the four types of characterization currently used, in-process characterization presents the most potential for improvement and cost savings. Current methods for in-process characterization usually require cessation of work to allow for radiation surveys to assess the rate of decontamination. Integrating radiation sensors with a decontamination technology would allow for in-process or online measurement of the progress of decontamination. Since present methods do not use in-process evaluations for the progress of decontamination, they may allow for "over-removal" of materials (removal of contaminated and non-contaminated materials together) as well as under-removal of material (not enough contaminated material has been removed). Over-removal increases the volume of radioactive waste to be disposed of and therefore disposal costs. On the other hand, under-removal will increase the exposure of the $D \& D$ worker to radiation as well as increase the cost since multiple surveys are required until all the contamination has been removed. Integrating technologies would facilitate the removal of only the contaminated concrete and reduce the total volume of radioactive waste to be disposed of. This project aims to minimize these problems.

The accurate characterization of contaminants is a critical task during several different phases of deactivation and decommissioning (D\&D) operations. The characterization process can be divided into four major categories: 1) safety characterization, wherein the contaminated structure is assayed for worker health and safety concerns; 2) initial site characterization, in which the identity of the contaminants and the extent of the contamination are determined in order to facilitate the remediation planning process; 3) in-process characterization, which is used to evaluate the efficacy of ongoing decommissioning activities; and 4) characterization performed for material disposition. Depending on the nature of the contaminants and the characterization objectives for a given remediation, the degree of sampling and analysis required for D\&D can significantly increase remediation costs.

This project focuses directly on the third characterization category, in-process characterization. Present characterization technologies typically require the cessation of decontamination activities, while the contamination removed and/or remaining is assessed. This usually requires the decontamination technicians to cease work and wait for the health physics technicians to perform a separate radiological survey. The specific aim of this subtask is to find in-process characterization methods, especially in the area of radiation sensors, that can be integrated with a suitable decontamination technology, in order to combine decontamination and characterization activities. The technology integration will also include data collection, storage and transmission components on the instrument for remote monitoring, and computer downloading functions.

A decontamination instrument with characterization and data collection technologies incorporated into it would allow for continuous decontamination activities, coupled by a realtime assessment of the amount of contamination removed and/or the amount remaining. The result would be a significant gain in productivity accompanied by cost and time savings. 
One of the key objectives of this subtask is to adapt an existing decontamination technology with commercially available characterization technologies to develop a prototype instrument that will be assessed and then commercially deployed. A closed-system decontamination technology will be selected that utilizes a vacuum or contaminant collection system and will be integrated with appropriate radiation sensing devices and data collection components. This integration of technologies will yield an improved instrument that may be continuously operated, removing contaminated materials and simultaneously assessing the removal progress.

This project is being carried out over a three-year period. The activities delineated for the first year will include commercial technology searches, component selection, and design. The subsequent years will include detailed integration design, unit testing, and commercialization. 


\subsection{CONCLUSIONS}

In order to determine if the sensor technology and the decontamination technology will face problems once integrated, a feasibility study (see Appendix B) was produced in which the effect of motion on the efficiency of a radiation sensor was measured. It was found that the effect is not negligible; however, it is not catastrophic, and if the sensors are properly calibrated, this obstacle can be overcome.

During the first year of this project, many important tasks have been accomplished. The search for radiation sensors provided knowledge on the technologies commercially available. This, in turn, allowed for a proper assessment of the properties, limitations, different methods of measurement, and requirements of a large number of sensors. The best possible characterization and data collection instrument and decontamination technologies were chosen using the requirement information in Appendix A. There are technical problems with installing sensors within the blasting head, such as steel shot and dust interference. Therefore, the sensor array is placed so that it will measure the radioactivity after the blasting. Sensors are rather sensitive, and therefore it is not feasible to place the sensor windows in such an abrasive environment.

Other factors, such as the need for radiation hardening in extreme cases, and the possible interference of gamma rays with the radio frequency modem, have been considered. These factors are expected to be negligible and can be revisited at the time of prototype production.

Factors that need to be addressed are the vibrations of the blasting unit and how to isolate the sensor array from these. In addition, an electromagnetic survey must be performed to ensure there will be no interference with the electronic component that will be integrated.

The integration design is shown in section 4.0. 


\subsection{PROJECT DESCRIPTION}

This project entails the following general technical objectives:

1. Delineate the requirements for the final integrated unit.

2. Evaluate decontamination technologies and generate a short list of technologies suitable for sensor integration.

3. Investigate commercially available radiation sensors and select a subset of sensors recommended for integration.

4. Conduct a technical review of the decontamination technology short list and select a final technology suitable for integration.

5. First-round design of sensor array and integration

6. First-round design of data collection, storage, and transmission system

7. First-round integration design.

The decontamination technology chosen will be selected from existing technologies that have a vacuum or contaminant collection system. The sensors and data collection/storage/transmission components will then be evaluated for placement within the integrated unit. There are several different options for placement of the radiation sensing devices. Based on FIU-HCET investigations, the sensors may be designed to integrate into only one, or into several locations. The ultimate goal for the placement of the sensors and data collection/storage/transmission components is to create an instrument that measures radiation and contaminant removal as "real time" as possible. Note that objectives 5, 6, and 7 are scheduled to be met in FY99. 


\subsection{RESULTS}

For the first year of this project, the following tasks have been accomplished:

\section{Delineation of the requirements of the final integrated unit.}

This task has been accomplished, and a document, which discusses the results in detail, has been produced. This document is included in Appendix A. The main points that were considered were the following:

- The environment in which the unit must be able to operate: The unit must be able to operate in radioactive environments.

- DOE-specified requirements for cleanup of surface contamination: The unit must be able to meet the requirements imposed by DOE.

- Cost requirements: The cost of integration should not exceed the original unit.

- Integration requirements: All instruments/technologies that will be integrated must physically fit on or within the decontamination unit without hindering performance.

- Decontamination technology needs: A good candidate would be an efficient concrete removal technology which also shows potential for compatibility.

2. Evaluation of decontamination technologies and generation of a short list of technologies suitable for integration.

This task has been accomplished and a technology has been selected. The set of vendors that were considered is the following: 
Table 1.

Decontamination Technologies Evaluated

\begin{tabular}{|c|c|c|c|}
\hline Company Namo & $\begin{array}{l}\text { General technologyjut } \\
\text { description }\end{array}$ & Techinologydescrption & Tochiologyname \\
\hline Va-Tran Systems, Inc. & Decontamination-Blasting & $\begin{array}{l}\mathrm{CO} 2 \text { pellet blasting; } \mathrm{CO} 2 \text { pellets } \\
\text { are shot against a surface where } \\
\text { impact and thermal shock crack and } \\
\text { remove layers of the surface. }\end{array}$ & SNO GUN \\
\hline $\begin{array}{l}\text { Oceaneering } \\
\text { International, Inc. }\end{array}$ & Decontamination-Emerging & $\begin{array}{l}\mathrm{CO} 2 \text { Blasting; Same as } \mathrm{CO} 2 \text { pellet } \\
\text { blasting but remotely operated. }\end{array}$ & ROVCO2 \\
\hline Cryogenesis & Decontamination-Blasting & $\begin{array}{l}\mathrm{CO} 2 \text { pellet blasting; } \mathrm{CO} 2 \text { pellets } \\
\text { are shot against a surface where } \\
\text { impact and thermal shock crack and } \\
\text { remove layers of the surface. }\end{array}$ & Cryogenesis \\
\hline IceSolv (2) & Decontamination-Blasting & $\begin{array}{l}\text { High pressure water; High pressure } \\
\text { water is propelled against surface. }\end{array}$ & $10 \mathrm{~K}$ Hydrolazer \\
\hline Unique Systems & Decontamination-Mechanical & $\begin{array}{l}\text { Scarification; Hand held unit which } \\
\text { utilizes attached scarification media } \\
\text { to remove surface coating. }\end{array}$ & Peena Cleaner \\
\hline Pentek Inc. (1) & Decontamination-Mechanical & $\begin{array}{l}\text { Scarification; Hand held unit which } \\
\text { utilizes attached scarification medial } \\
\text { to remove surface coating. }\end{array}$ & Roto-Peen Scaler \\
\hline Pentek Inc. (2) & Decontamination-Mechanical & $\begin{array}{l}\text { Scarification; Mechanical methods } \\
\text { are used in order to abrade or } \\
\text { fracture surface. }\end{array}$ & $\begin{array}{l}\text { Corner cutter, Squirrell I, } \\
\text { Squirrell II }\end{array}$ \\
\hline $\begin{array}{l}\text { Surface Technology } \\
\text { Systems }\end{array}$ & Decontamination-Blasting & $\begin{array}{l}\text { Sponge Blasting; Surface is blasted } \\
\text { with different grades of sponges. }\end{array}$ & $\begin{array}{l}\text { Advanced Recyclable Media } \\
\text { System (ARMS) }\end{array}$ \\
\hline LTC Americas (2) & Decontamination-Blasting & $\begin{array}{l}\text { Grit blasting; Abrasive materials } \\
\text { are projected against the surface } \\
\text { using compressed air. }\end{array}$ & LTC 1073 \\
\hline $\begin{array}{l}\text { Textron Systems } \\
\text { Division }\end{array}$ & Decontamination-Mechanical & $\begin{array}{l}\text { Scarification; Electric current is } \\
\text { passed through water by electrodes } \\
\text { which cracks surface. }\end{array}$ & $\begin{array}{l}\text { Electro-Hydralic Scabbling } \\
\text { System (EHS) }\end{array}$ \\
\hline Hotsym & Decontamination-Mechanical & $\begin{array}{l}\text { Hot water Flushing; Hot water is } \\
\text { used to dissolve chemical species or } \\
\text { flushing loose debris. }\end{array}$ & Model 550B \\
\hline $\begin{array}{l}\text { P.W. Stevens } \\
\text { Environmental Co. }\end{array}$ & Decontamination-Mechanical & $\begin{array}{l}\text { Uitra high pressure water, High } \\
\text { water pressure is used to remove } \\
\text { concrete. This technology is similar } \\
\text { to hydroblasting. }\end{array}$ & UHP Flow International \\
\hline $\begin{array}{l}\text { Pegasus International, } \\
\text { Inc. (4) }\end{array}$ & Decontamination-Blasting & $\begin{array}{l}\text { Steel abrasive blasting; Steel shot is } \\
\text { projected against surface using } \\
\text { compressed air. }\end{array}$ & $\begin{array}{l}\text { NELCO Porta Shot Blast } \\
\text { Model EC-7-2 }\end{array}$ \\
\hline AEA O'Donell & Decontamination-Blasting & $\begin{array}{l}\text { Sponge blasting; Surface is blasted } \\
\text { with different grades of sponges. }\end{array}$ & STS Sponge Jet \\
\hline A.R.C. & Decontamination-Blasting & $\begin{array}{l}\text { Wet ice blasting; Low pressure air } \\
\text { is used to project wet ice. }\end{array}$ & Iceblast \\
\hline
\end{tabular}


Table 1.

Decontamination Technologies Evaluated (continued)

\begin{tabular}{|c|c|c|c|}
\hline $\begin{array}{l}\text { Pegasus International, } \\
\text { Inc. (2) }\end{array}$ & Decontamination-Blasting & $\begin{array}{l}\text { Shot blasting; Steel shot is } \\
\text { projected against surface using } \\
\text { compressed air. }\end{array}$ & $\begin{array}{l}\text { NELCO Porta Shot Blast } \\
\text { Model JHJ-2000 }\end{array}$ \\
\hline $\mathrm{J} \& \mathrm{~B} /$ Concrete cleaning & Decontamination-Blasting & $\begin{array}{l}\text { Grit blasting-steel grit; Steel grits } \\
\text { are projected against the surface } \\
\text { using compressed air. }\end{array}$ & Blasttrac \\
\hline Barlett & Decontamination-Blasting & $\begin{array}{l}\text { Plastic blasting; Plastic media shot } \\
\text { against surface. }\end{array}$ & Plasblast \\
\hline Church \& Dwight & Decontamination-Blasting & $\begin{array}{l}\text { Sodium bicarbonate blasting; Low } \\
\text { pressure air is used to propel } \\
\text { carbonated water. }\end{array}$ & Armex \\
\hline $\begin{array}{l}\text { Pegasus International } \\
\text { Inc. }\end{array}$ & Decontamination-Mechanical & $\begin{array}{l}\text { Shotblasting; steel shot is blasted } \\
\text { against surface in order to } \\
\text { abrasively remove concrete. }\end{array}$ & EVE 250 VHC shot blasting \\
\hline ARAMSCO Company & Portable Shot Blasting unit & $\begin{array}{l}\text { Shot blasting; Steel shot is } \\
\text { projected against surface using } \\
\text { compressed air. }\end{array}$ & BLASTRAC \\
\hline $\begin{array}{l}\text { Apheus Cleaning } \\
\text { technologies Corp. }\end{array}$ & Decontamination-Mechanical & $\begin{array}{l}\mathrm{CO} 2 \text { pellet blasting; } \mathrm{CO} 2 \text { pellets } \\
\text { are shot against a surface where } \\
\text { impact and thermal shock crack and } \\
\text { remove layers of the surface. }\end{array}$ & $\begin{array}{l}\mathrm{CO} 2 \text { MiniBlast SDO-5, CO2 } \\
\text { CleanBlast, } \mathrm{CO} 2 \text { BlockMaster, } \\
\text { and } \mathrm{CO} 2 \text { WhisperBlast } 127\end{array}$ \\
\hline $\begin{array}{l}\text { Contam-Away Blasting } \\
\text { Systems }\end{array}$ & Decontamination-Blasting & $\begin{array}{l}\text { Sodium bicarbonate blasting; Low } \\
\text { pressure air is used to propel } \\
\text { carbonated water. }\end{array}$ & Model 100CS \\
\hline
\end{tabular}

\section{Investigation of radiation sensors and selection of a subset recommended for integration.}

There was a large search for commercially available radiation sensors and some of these were selected for consideration. The selection criteria is delineated within Appendix A. This task was completed, and the sensors are summarized below:

1. Abacus by Chemrad: an array of five sensors, $100 \mathrm{~cm}$ squared, to detect the various types of radiation, mounted in one housing with accompanying electronics unit, battery power, and GPS. Current usage is for static situations. Proposed situation is for dynamic (in motion) measurements.

2. Beta Gamma Sensor: connected to a GPS and data-logger. The system would measure beta and gamma radiation in the field, while recording the coordinate position.

3. Phoswich: these types of sensors measure alpha, beta, and gamma radiation simultaneously through use of scintillating crystals and photomultiplier tubes. The idea would be to incorporate a detector in the exit line of a floor decontamination unit or at or near the blast head of the same unit. The latter is preferred due to the high speed at which particulates travel through the exit line. 
4. Radstar by SAIC: a small personal dosimeter that sends real-time data back from the unit worn by workers to a computer via RF transmitters. The sensors would need to be adapted for $\mathrm{dpm}$ readouts instead of dose rates.

5. Victoreen GM detector, Model No. 05-783: detects alpha above $4 \mathrm{MeV}$, beta above $70 \mathrm{keV}$, and gamma above $6 \mathrm{keV}$.

6. Rados Universal Survey Meter RDS-120: advanced dose rate meter with two built-in GM tubes. Data logging features for cps values and data output via RS-232.

7. Rados Multi-purpose Survey Meter RDS-110: measures beta, gamma, and x-ray. Contains one energy-compensated GM tube controlled by a microprocessor. Can attach external beta or gamma probe for added sensitivity.

8. NRC Radiac Set, AN/PDR-77: detects alpha, beta, gamma, and x-ray and comes with headphones for audible signals. Designed for military operations and detects Plutonium under adverse weather conditions.

9. NE Technology Delta 5 Advanced multi-purpose ratemeter: portable alpha/beta meter with attachment for headphones and PC interface.

10. Bicron Electra microprocessor-based ratemeter: measures alpha/beta and has attachments for plastic scintillation detectors and energy-compensated GM probes, has alarm settings, and is portable.

11. Bicron PRM510 Portable radiation monitor: lightweight ratemeter, $20 \mathrm{~cm}^{2}$ window, detects alphas, betas, and gammas and is portable with alarms.

12. Bicron $600 \mathrm{~cm}^{2}$ Scintillation probe, DP8: has stainless steel mesh grill for extra window protection, measures alphas and betas using dual channel ratemeters. Has field replaceable thin radiation windows, high surface area and low weight.

13. Thermo Nutech LARADS: a portable probe that measures radiation and sends it back to a computer for mapping of areas. The advantage is it is calibrated for dynamic measurements and provides positioning and mapping data. The exact types of radiation need to be measured.

14. Thermo Nutech GPRS-II: GPS/radiation sensor system for performing outdoor surveys.

15. Thermo Nutech Segmented Gate System (SGS): a conveyor belt system having a detector array for measuring alpha, beta, and gamma in a dynamic setting. Array is proprietary, will need to investigate the possibility of buying it from them or disclosing the details of the design. Advantage is that the system is dynamic and the radiation measurements are made from a platform adjustable in height (the distance from the material is varied, which is identical to our application).

16. Beta Scintillation fiber optics: fiber optic elements that sense beta in a real-time application. Advantage would be the small size and ease of integration. Processing electronics and hardware also required.

17. SRA surface contamination monitor by Shonka: measures alpha and beta in a dynamic setting and maps the results. Is already designed to be mobile/portable. 


\section{Technical review of the decontamination technology short list and selection of a final technology suitable for integration.}

To comply with the requirements delineated at the onset of the project and presented in Appendix A, a floor decontamination technology was selected. The technology selected is a shot blasting unit, EBE 350. This technology is a floor shot blasting unit that works in combination with a vacuum unit in order to gather the waste produced. A picture of the unit is presented in Figure 4.1.The reasoning was first simplicity in its approach as compared with the wall decontamination units. Also, the floor decontamination systems are able to produce higher production rates than the vertical technologies.

Mechanical abrasion was chosen as the removal method due to its efficiency in containing waste materials and its ability to remove substantial amounts of concrete (typically, up to 1/4") as opposed to only removing coatings or top layers. Other types of decontamination units were eliminated from the selection process due to their method of removal or waste collection method. Laser removal methods were not chosen due to their limitation of only removing superrficial layers and with inconsistent success.

Other types of technologies were also eliminated from the selection process due to their uncontrolled generation of airborne contaminants. For example, ice and water blasting were not chosen since they both generate large amounts of airborne particulate/water vapor/contaminants. Any such technology will have a limited application at DOE facilities and is therefore not consistent with that primary goal of commercializing the integrated technologies.

In conclusion, centrifugal shot blast technology was selected for its high production rate, portability, contaminant containment, and integration potential. The units are small enough to be portable and big enough to allow incorporation of new components. The resulting technology integration will produce a very useable technology with real-time assessments of radiation.

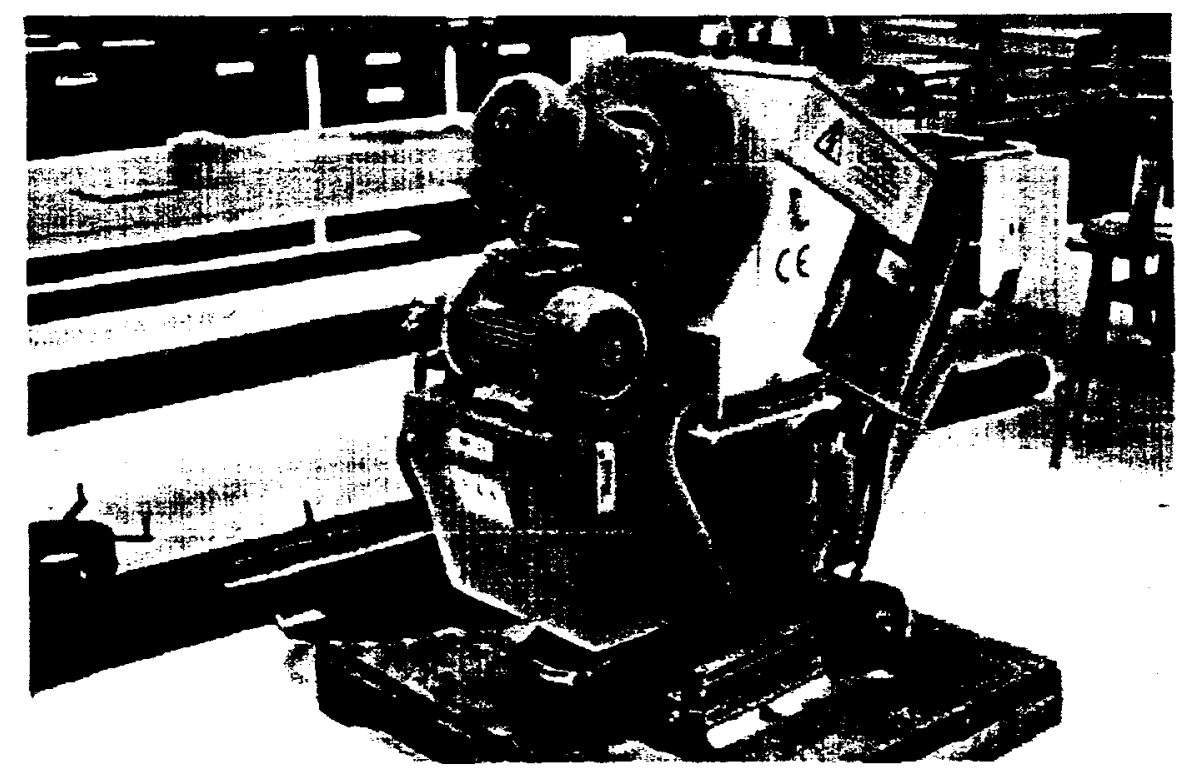

Figure 1. European Blasting Equipment 350 Centrifugal shot-blasting unit. 


\section{First round design of sensor array and integration (vacuum line integration, instrument inlet integration, separate unit integration, or a combination).}

Through several peer discussions and some vendor interface, it was decided that the two most promising places for placement of the sensors would be in 1) exit hose and 2) close to the blasting surface.

The sensors on the exit line would have readings directly proportional to the contamination level of the waste within the hose, thereby providing information that could be used to determine the progress of decontamination. It was then found, as more quantitative results were desired, that there are several problems with this approach, which may include the possibility of a buildup of contamination within the hose.

Another problem with the exit hose is that quantitative correlation between the readings obtained by a sensor on the exit hose and the counts per unit area of the concrete surface are difficult to produce. Since the blasting unit does not produce a completely smooth surface and since it is difficult to track the exact spot where the dust in the hose at that precise moment originated, this approach is less appealing than originally thought. However, FIU-HCET will continue to pursue this task.

On the other hand, positioning the sensors behind the blasting unit provided a good solution for the technical objectives, considering the technical limitations. The sensors behind the blasting unit would not encounter a stream superfluous in abrasive material, this way reducing the risk of damage to the sensors. In addition, reliable data can be taken in this environment without having to compensate for interference due to dust or the abrasive media. An array of sensors can be designed so that it is vibrationally isolated and so that it has the appropriate distance from the surface to prevent contact. Note that contact with the surface could possibly damage the sensors, for the blasting unit does not produce an even, smooth surface. Peaks and burrows are produced on the surface, and dragging the sensors against this surface would damage them. These ideas will be expanded where the conceptual design of the integrated unit is discussed. This task will also continue during FY99.

A sensor array that meets the needs of this project is commercially available. A commercial vendor (CHEMRAD) was selected, and a contract was put in place with this vendor. A list of items included in this purchase order is provided below along with the terms for this contract. In addition, a detailed discussion of the feasibility to integrate charaterization technologies is included in Appendix B.

The required items were listed as follows:

1. Field Controller Unit (FCU): The FCU contains the custom computer board with power supply to interface to the radiation detector array.

2. STEP Engine: The STEP engine includes the ultrasonic crystal driver board and timing circuitry for the conduct of STEP positioning at $1 \mathrm{~Hz}$.

3. Palm Top Option: The Palm Top options allow for control and/or display of the survey/mapping results at the surveyor/scabbler location. 
4. BP/Mounting Option: Mounting fixtures for mounting the FCU/1-STEP package on a Back Pack frame or on the Scabbler (by others).

5. RCC option: The Remote Control Center RF link from the FCU to the master controller with a serial cable for connecting to the RCC PC (by others).

6. RCC PC/Printer Option: It is understood that FIU-HCET will supply a suitable PC with printer for testing and delivery.

7. Logger Analyze is the core software for post survey analysis of survey data.

8. The Rad Module is the add-in to Logger Analyze to support radiation detectors.

9. The INRADS Module is the add-in to Logger Analyze to support analysis of 1-STEP data.

10. Three (3) Ludlum 44-10 $2 \times 2$ sodium iodide ( Nal) detectors will be supplied to monitor the gamma component of any surface contamination.

11. Two (2) Ludlum 43-99 gas proportional detectors. Each is approximately 5.75 " by 17.75 " with $4 \sim 100 \mathrm{~cm}^{2}$ active area cells. Each will be supplied and configured as desired to monitor the alpha and/or beta component of any near surface contamination. The optimum configuration and operation of these detectors will be determined during development and testing.

12. A Ludlum 440-12W with individual power supplies and pre-amps for up to 12 different detectors wil be supplied.

13. The associated cables for connecting the Ludlum 440-12 to the detectors.

14. A 12-volt DC battery for providing power to the Ludlum and CHEMRAD electronics.

15. A regulator for the $\mathrm{P}-10$ gas.

16. P-10 bottle and gas (by others).

17-20. An allowance for designing and building a prototype detector frame, mounting the detector array, integrating, and testing the integrated system is included.

21. Eight hours of consulting time for the Principal Developer and the Principal Health Physicist are included herein.

\section{First-round design of data collection, storage and transmission system.}

A search for candidate items was conducted and a folder containing FIU-HCET findings was compiled. This folder included documents HCET-C005-55-23 to HCET-C005-060-23 A conceptual design was developed based on these findings. This conceptual design is shown schematically on Figure 2. It was found that the sensor array vendor could provide a system that matched the conceptual design drafted, this way integrating the whole system. In addition, its data logger analogue has open channels that can be used to connect additional sensors, i.e., positioning system. The option for remote transmission is quite viable; however, end-users have shown little interest in it. Therefore, this option must be maintained open for remotely operated units, but it will not be permanently attached to the integrated system, this way allowing for remote transmission when necessary. 


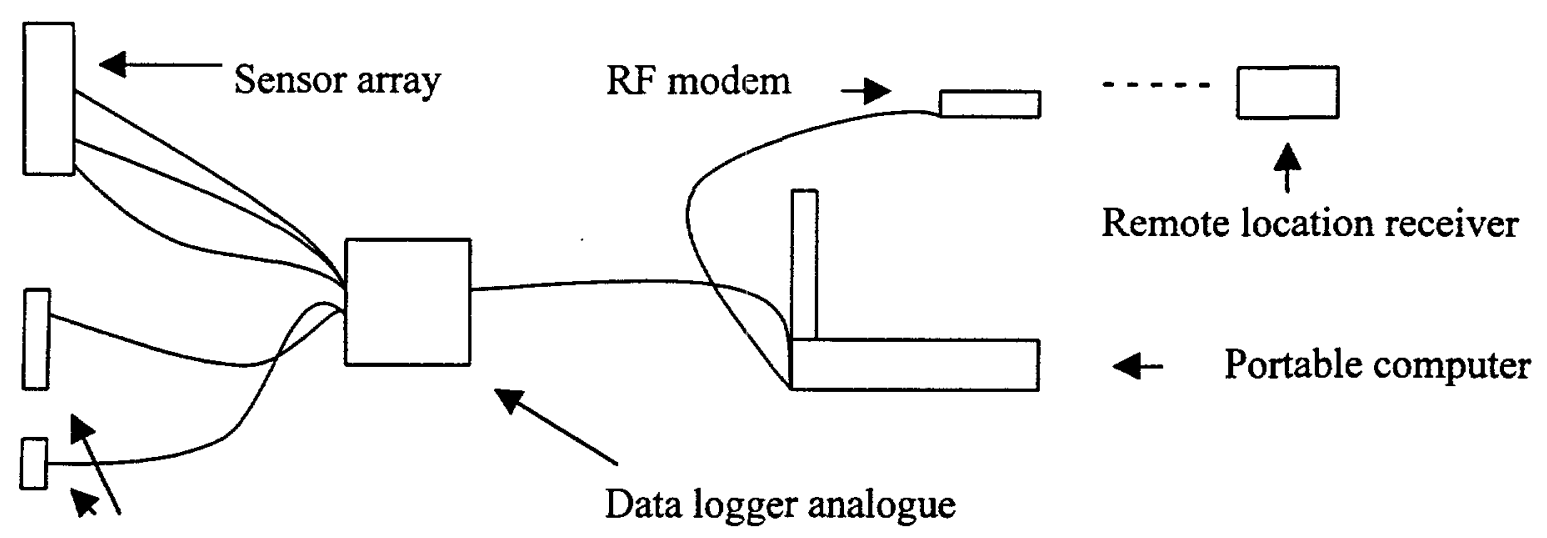

Assorted sensors

Figure 2. Schematic of conceptual design.

In addition, a feasibility study was performed in order to determine the efficiency loss in radiation sensors due to motion parallel to the target surface. It was found that the effects were significant; however, these can be accounted for by calibrating the instrument or the electronics box (data-logger analog). The results are included at the end of this section.

\section{The first round design of the fully integrated unit}

The conceptual design can be seen in Figure 2. Figure 3 is a drawing that details important regions of the blasting unit. Dimensions for these sections are included; however, features such as shape of motors and electrical wires have not been included. The sensor array, the portable computer, calibration gas chamber, and battery are all included in this drawing.

Several factors were discussed during the design phase. First of all, the issue of positioning the sensors in the best suitable place brought up several interesting points:

1. The issue of positioning the sensors in the best suitable place brought up several design challenges.

A. The sensors are fragile. The blasting head is the space where the blasting occurs. Shot and concrete particles/dust occupy this space. The speed of the blasting media is high and so is the speed of the concrete particles. No rugged sensor that could stand the environment inside the blasting head was found. In fact, it was found that most sensors had rather sensitive windows. For that reason, the shot-blasting head was not a good location for these sensors. The next best choice is where they are positioned in the drawing-after the decontamination unit. Here they are out of the range of the shot, especially if additional brushes are placed on the unit, and they can still provide an accurate assessment of the contamination below. 


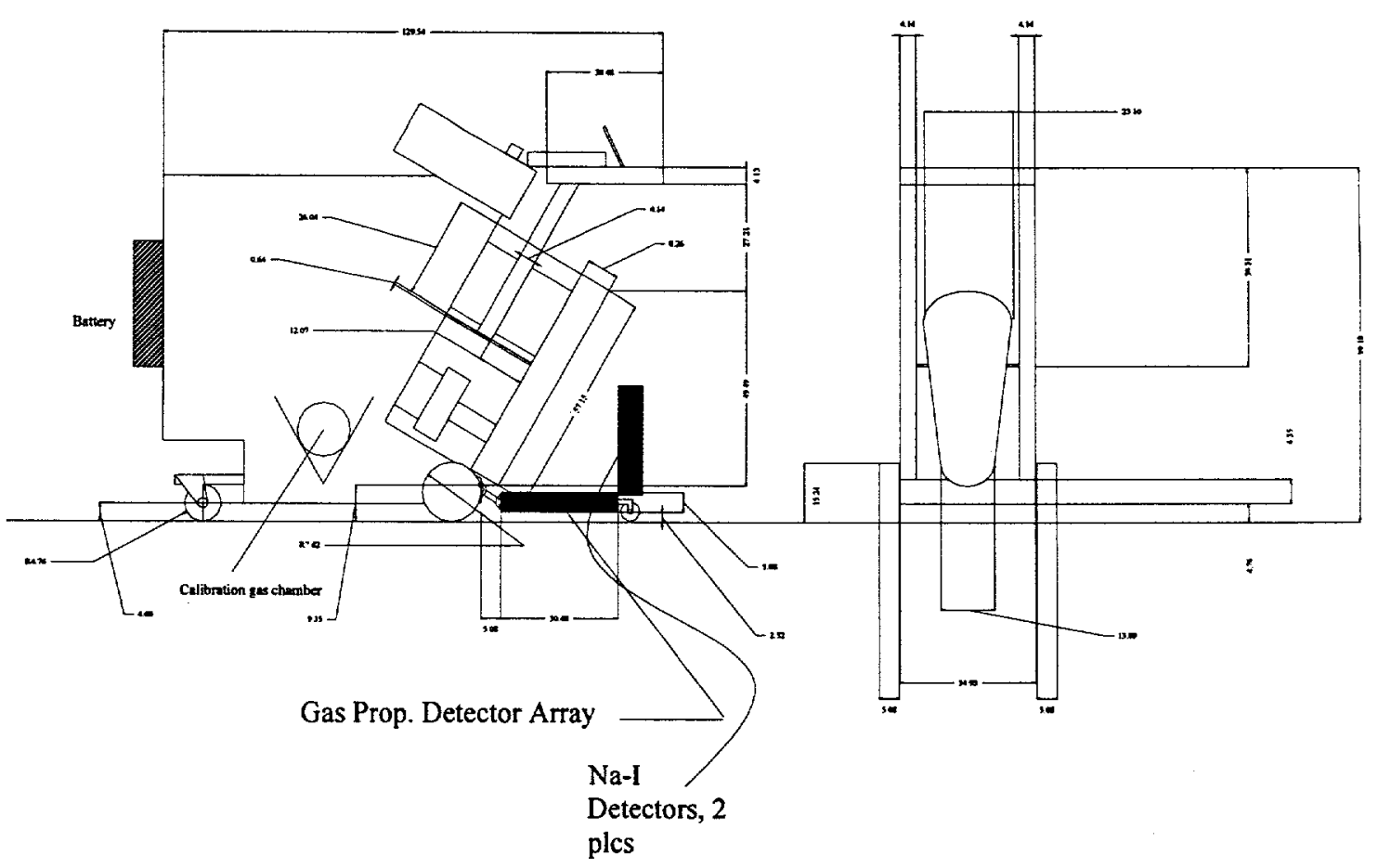

Figure 3. Conceptual drawing of integrated system.

B. Reading interference. Concrete particles and shot would interfere with the readings. Alpha particles, for example, interact with matter, and as they collide with air particles, they lose energy until they lose their charge and their energy. This happens over a short range in air. In an environment with a fluid of larger density (air with dust and steel shot), this effect is more pronounced. Obviously, if a sheet of paper can stop alpha radiation, a steel shot or concrete particles can also do the same, reducing the number of alpha particles that could penetrate over a short range. It should be noted that in most cases, the number of alpha particles that penetrate past a layer (1-2") of this fluid would exponentially decay as a function of the distance. There may be some possibility of calibrating, in the rare case that some alpha particles manage to go through, in order to account for this effect; however, it is not a steady system. The speed of the fluid is not completely constant, the density is not constant either, and other factors, such as contamination build-up in the parts of the unit can make calibration in this environment much less practical than simply taking the readings immediately after blasting.

C. Sensor Array. The sensor array is designed so different sensors can be used. The sensors are not permanently placed in position. They are removable in order to allow the users to use the sensors that best fit their needs.

2. An interesting point that also was brought up is that remote reporting of the data was not necessary in all cases. End users did not show interest in applications where the unit could be operated manually. The logic was that the operator should be able to decide from the readings whether the area is decontaminated or not, therefore leaving no need for remote 
replication of the data, especially since the data is stored for future reference. There are, however, applications where the unit would have to be remotely operated and where there would be no operator to monitor the data. For that reason, the option has been left open, but it was not included in the drawing above.

3. The portable computer was placed in an area of high visibility, this way allowing the operator to monitor the readings continuously.

4. A feasibility study for the efficiency loss of rad sensor due to motion was performed, and the conclusions are included in Appendix C. 


\subsection{ACTIVITIES PLANNED FOR FY99}

The following tasks are planned for fiscal year 1999:

Task 1. Translate conceptual design documents to production specifications.

Design documents, such as CAD drawings, assembly drawings, and integration design drawings, will be converted to specifications that can be used by commercial manufacturers to build and assemble a prototype unit. All drawings and documents will be maintained electronically and/or in hard copy, and a quality control system will be used to ensure the preservation and quality of said documents.

Task 2. Complete the deployment plan.

The deployment plan, begun in FY98, will be finalized during FY99. The plan will include DOE sites willing to test the integrated unit and specific names and contacts for testing and deployment activities. The plan will also contain information relevant to commercialization and allow for easy dovetailing with the commercialization plan to be developed in the third year of the project.

\section{Task 3. Generate/produce an operational prototype.}

Using the production specifications listed above, a prototype unit will be manufactured. This will involve the manufacture of new components and the integration of existing commercial components. It is probable that multiple manufacturing/assembly sources will be used, including FIU-HCET engineering personnel.

\section{Task 4. Generate demonstration plan at FIU-HCET.}

A demonstration plan will be generated to perform an in-house evaluation of the constructed prototype unit. The test plan will contain protocols for operation and evaluation, quality control procedures, calibration procedures, documentation procedures, and procedures for resolving engineering-related issues. The demonstration will also include the participation of all involved stakeholders (DOE, commercial companies, etc.).

\section{Task 5. Perform FIU HCET demonstration.}

The test plan generated in the previous step will be implemented at the HCET testing facilities to evaluate the performance of the integrated unit. The tasks below address the procedures for posttesting activities and collection and storage of testing data.

\section{Task 6. Document FIU-HCET demonstration.}

All testing performed at FIU-HCET will be conducted according to strict quality control procedures (specified in internal FIU-HCET documents). Logbooks with dated and signed pages will be used; testing personnel will be trained in documentation and testing procedures; any deviations from baseline will be duly recorded; all records will be kept under strict control by the project manager; etc. In summary, the following elements will be documented regarding the FIUHCET testing scenario: 
- Setup and operation of the prototype unit

- General observations

- Description of the tests performed

- Description of calibration procedures

- Description of prototype performance

- Description of personnel involved in the project and the training administered

- Description of any anomalies or malfunctions

- Description of any recommendations for design modifications and/or improvements

- Description of the general environmental conditions of the test scenarios.

\section{Task 7. Perform a cost-benefit analysis.}

After the prototype has been successfully tested at the FIU-HCET facilities, a cost-benefit analysis will be performed to determine the commercial potential for the technology. All future costs and benefits will be estimated and converted to a present value (present value analysis). A rate of interest consistent with technology commercialization will be selected (versus an interest rate typically used for government investments) for the analyses, and a five-year time frame will be selected for the analysis. If the cost-benefit analysis indicates that commercialization of the technology is not a feasible option, the scope of the project will be re-examined and adjusted accordingly.

\section{Task 8. Perform and document engineering design changes.}

Any design changes generated as a result of testing or design review will be thoroughly documented using strict quality control procedures. Any design changes that result from the FIUHCET testing scenario will be recorded under strict quality control measures, and the changes will be implemented (via drawing modifications, document modifications, refabrication, etc.).

Task 9. Remanufacture any modified equipment.

Equipment modified as a result of the previous tasks will be refabricated using modified design/production/assembly drawings or related engineering information. This may involve multiple commercial manufacturers and/or FIU-HCET personnel.

Task 10. Re-assemble the prototype.

Once the prototype and all its components have been modified to satisfy the objectives of the project, the prototype unit will be re-assembled on-site at FIU-HCET facilities.

\section{Task 11. Perform in-house verification of the design changes.}

After re-assembling the prototype, an engineering review will be performed to botn verify and validate the objectives of the project and of the integrated unit. The verification will be thoroughly documented using in-house quality control measures. 


\section{Task 12. Generate detailed deployment work plan.}

A deployment work plan will be generated for the testing and evaluation of the prototype unit at a DOE facility. The plan will be generated in cooperation with the facility at which the testing will take place and will include references to said facility's procedures and protocols. The plan will delineate personnel and their respective responsibilities, the test scenarios that will be carried out, how they will be carried out, mobilization and demobilization procedures, decontamination procedures, testing documentation (written, photo, and video), "in the field" design modifications, and any other issues relevant to the testing.

\section{Task 13. Implement deployment work plan.}

The deployment work plan generated in the previous task will be implemented at a DOE site, in accordance with the deployment plan developed as part of the same project.

\section{Task 14. Document results of the deployment.}

All pertinent information regarding the testing scenario carried out at the DOE facility will be recorded in a fashion similar to that for documenting HCET testing scenarios.

\section{Task 15. Perform and document engineering design changes.}

Engineering design changes will be recommended, documented, and carried out, as applicable, according to the results of the DOE testing scenarios, in a manner similar to that previously described for FIU-HCET testing scenarios.

\section{Task 16: Prepare Year-End Report.}

The Year-End Report will be prepared, providing a summary of the tasks performed during FY99 and their results along with a brief description of the tasks performed during FY98. 


\subsection{LESSONS LEARNED}

Throughout this project, some interesting items have been discovered that are worth mentioning. First of all, it should be noted that staff with a wide range of expertise was quite useful. This is simply because in these technology integration projects, a good foundation in the areas of electrical and mechanical engineering, field experience, as well as a good understanding of the basic functioning of radiation detectors allowed FIU-HCET to tackle this problem. Access to FIU-HCET decommissioning and decontamination databases on D\&D technology also was quite useful.

In addition, close contact with vendors helped to fully assess the potential of their products. This was found true for the radiation sensors where several different units and methods of detection were found. Also, the number of possible vendors was quite large, and lots of filtering has to be done in order to determine potential vendors for the sensors. 


\section{APPENDIX A}

ONLINE MEASUREMENT OF THE PROGRESS OF DECONTAMINATION:

REQUIREMENTS DOCUMENT 
The document that follows delineates the design requirements for the Online Monitoring of the Progress of Decontamination. The intent of this document is to provide a baseline "needs statement" which will subsequently steer the efforts of the project. Since the project is specified to cover a span of three years, the current document is intended to only address the first year's worth of activities.

The present document is intended to be a "living document" and will be revised accordingly during the length of the project. All attempts will be made to make the revisions only at key task/subtask completion points and/or milestone points. Revisions will be noted in the bottom left corner of each page.

\section{GENERAL DESCRIPTION OF PROJECT AND NEED FOR AN INTEGRATED UNIT}

The accurate characterization of contaminants is a critical task during several different phases of deactivation and decommissioning (D\&D) operations. The characterization process can be divided into four major categories: 1) safety characterization, wherein the contaminated structure is assayed for worker health and safety concerns; 2) initial site characterization, in which the identity of the contaminants and the extent of contamination are determined in order to facilitate the remediation planning process; 3 ) in-process characterization, which is used to evaluate the efficacy of ongoing decommissioning activities; and 4) characterization performed for material disposition. Depending on the nature of the contaminants and the characterization objectives for a given remediation, the degree of sampling and analyses required for D\&D can significantly increase remediation costs.

This subtask focuses directly on the third characterization category, in-process characterization. Present characterization technologies typically require the cessation of decontamination activities, while the contamination removed and/or remaining is assessed. This usually requires the decontamination technicians to cease work and wait for the health physics technicians to perform a separate radiological survey. The specific aim of this subtask is to find in-process characterization methods, especially in the area of radiation sensors that can be integrated with a suitable decontamination technology, in order to combine decontamination and characterization activities. The technology integration will also include data collection, storage and transmission components on the instrument for remote monitoring and computer downloading functions.

A decontamination instrument with characterization and data collection technologies incorporated into it would allow for continuous decontamination activities coupled by real-time assessments of the amount of contamination removed and/or the amount remaining. The result would be an overall gain in productivity accompanied by cost and time savings.

One of the key objectives of this subtask is to adapt an existing decontamination technology with commercially available characterization technologies to develop a prototype instrument that will be assessed and then commercially deployed. A closed-system decontamination technology will be selected that utilizes a vacuum or contaminant collection system and will be integrated with appropriate radiation sensing devices and data collection components. This integration of technologies will yield an improved instrument that may be continuously operated, removing contaminated materials and simultaneously assessing the removal progress. 
This project will be carried out over a three-year period. The activities delineated for the first year will include commercial technology searches, component selection, and design. The subsequent years will include detailed integration design, unit testing, and commercialization.

\section{ENVIRONMENTS IN WHICH THE INTEGRATED UNIT WILL BE USED}

The final integrated unit will be used primarily for concrete floor and wall decontamination. The environments will be considered radioactive and will have a variety of radioactive isotopes present. For example, consider the following excerpt from a report prepared for the Department of Energy, Idaho Operations Office:

\subsection{Contaminant Fixation Mechanisms}

Most contamination can be categorized into two broad levels: "loose" and "fixed". Loose contamination is characterized as dirt that is on the surface of an object and can be removed by wiping or flushing. It is loosely held and easily removed. Typically, loose contamination is contained either in dust or a fragile crust layer. Fixed contamination, on the other hand, is described as that contamination that is not easily removed by flushing or wiping, or not removed on a health Physics "smear" survey of a surface. This categorization is somewhat subjective, depending on how much force is put into the smear, wipe, or flush.

The removal of fixed contamination is of primary concern in decontamination work. Five subcategories of contamination mechanism are as follows: 1) reactor, or induced radioactivity from a neutron source; 2) abraded, 3) dried on surface; 4) acid-etched; and 5) trapped in an oxide layer on the surface of the metal. Activated rebar in a concrete wall of a reactor cavity is an example of reactor induced radioactivity. Abraded contamination is described as being ground into a surface, as with moving mechanical components where contamination is located.

During discussions with various personnel around the plant, abraded, dried, and acid-etched contamination were mentioned most often. Transfer from abrasion, or intimate contact with dry and wet contaminated items, is an obvious mechanism. It is typically estimated that $10 \%$ of the contaminants would be transferred simply by contact wit dry contaminated items. If abrasive action is added, greater transfer would be expected.

The same document also summarizes data compiled during the decontamination of CPP-601 first cycle process equipment. The primary contaminants found were $\mathrm{Zr} / \mathrm{Nb}-95, \mathrm{Ru}-106, \mathrm{Sb}-125$, Cs134,137 and Ce-144. The document continues with a description of how non-radioactive $\mathrm{Cs}$ and $\mathrm{Zr}$ salts were used as stand-ins in decontamination technology performance testing.

\section{DOE-SPECIFIED REQUIREMENTS FOR CLEANUP OF SURFACE CONTAMINATION}

In order to select appropriate radiation sensors and related integration components, it is first necessary to identify the cleanup levels for radiation which will be used in conjunction with the unit. For example, the lower the cleanup level, the lower the detection limit will need to be for the radiation sensors. 
DOE has specified surface contamination cleanup levels for a wide variety of radioactive contaminants in DOE Order \# 5400.5.

\section{COMMERCIALIZATION REQUIREMENTS}

The final goal of the integrated unit specified in this project is to have a commercially deployable unit. To that end, it is an initial requirement to select only technologies, or components, which are already commercially available and can be integrated with slight or reasonable modifications.

The decontamination technology will therefore be selected from a subset of technologies that are already being used successfully for the removal of contaminated concrete. The radiation sensors will be selected from existing radiation sensors which measure alpha, beta, and gamma radiation. Radiation sensors for the measurement of neutrons will not be addressed during the first year of the current project. If promising technologies for the real-time measurement of neutron radiation are found during the course of the project, they will be evaluated for possible inclusion in the second and third years of the project.

The data collection/storage/transmission data components will be selected from the already existing base of vendors. The components will be selected to perform in radioactive environments, and every attempt will be made to keep the system design as simple as possible.

Throughout the first year of work, the related vendors of the technologies chosen for integration will be consulted and included in the process. The intent is to assure that the vendors are willing to participate in the integration process and possibly to manufacture the final integrated units. The second and third years of the project will include recommendations to DOE as to the commercialization of the integrated unit.

\section{COST REQUIREMENTS}

There is currently little doubt that an integrated unit would represent a cost savings to DOE in their cleanup efforts. The financial requirements of this integration project are the following:

1. The total cost of integration does not exceed the savings that would be seen in the first five years of operation.

2. The cost of integration for each component is not in excess of the original cost of the component. For example, if a radiation sensor is considered, the costs associated with its integration (i.e., fabrication of special brackets, new calibration procedures, etc.) must not exceed the initial price of the sensor.

3. The cost of the remote monitoring station must not exceed the cost of the decontamination technology integrated with radiation sensors.

All cost estimates generated during the course of the first year of work will be "estimates" only. General project costs incurred by FIU-HCET will be included in the calculations. 


\section{INTEGRATION REQUIREMENTS}

The physical requirements for the integration are as follows:

1. The radiation sensors must fit physically in such a way as to not hamper normal operation of the decontamination technology. Their calibration processes may require initial calibration. If it is also deemed that in-process calibrations are required, every attempt will be made to keep the operation simple.

2. The data collection/storage/transmission hardware must also not inhibit normal operation of the decontamination technology. The same calibration constraints apply to that unit as to the radiation sensors.

3. Radiation Sensor design: The radiation sensors may be placed in three possible different locations; at the inlet to the decontamination technology (effector), in the exit pipe for contaminated materials, or in a separate unit to be used in conjunction with the decontamination technology. The final locations will be chosen during the sensor array design phase.

4. The final integrated unit must be portable and be capable of being moved from area to area within a DOE facility.

5. The remote monitoring station must be capable of receiving data at a minimum up to 20 feet away from the decontamination unit.

\section{DECONTAMINATION TECHNOLOGY NEEDS}

The general requirements for the decontamination technology that will be chosen are as follows:

- A concrete removal technology for floors and/or walls.

- Commercially available with a proven track record or reasonable expectation for success in operability.

- Vendor is willing to participate in an integration project.

- The technology can be adapted to incorporate radiation sensors and data collection/storage/transmission hardware.

- The unit is designed for work in radioactive environments.

- Waste collection system, i.e., vacuum system

- Close loop technology.

At this point in the project, a decontamination rate will not be specified (i.e., 50 square feet per hour) as a constraint. The intent is to focus on the quality of the final integrated project. The decontamination rate will however be taken into consideration and all attempts made to keep it as high as possible. 


\section{RADIATION SENSOR REQUIREMENTS}

The radiation sensors selected for this project must be capable of measuring alpha, beta, and gamma radiation. The first design round, which will be performed during the first of the three years, will be carried out with the goal of selecting radiation sensors with detection limits as close to the cleanup levels specified by DOE for surface contamination. If it is found that the detection limits of the most appropriate sensors are not capable of measuring down to the cleanup levels, an evaluation will be made as to their functionality. While the intent of the final integrated unit is to decrease cycle time of cleanups by being able to measure radioactivity in materials removed or being removed, it is also recognized that a final radiation survey performed by Health Physics personnel will still be necessary prior to "free release". Therefore, even if the radiation sensors do not measure accurately down to the free release criteria, the integrated unit will still be capable of greatly reducing cycle time.

As also previously stated, the radiation sensors must be selected from commercially available units. The vendors associated with these sensors will be required to participate in the integration process and will be consistently included in the design process.

The radiation sensors selected must also require a typical calibration process, as opposed to an elaborate one. This requirement will ensure that the final unit may be calibrated successfully in the field, without causing excessive delays or long startup times.

\section{DATA ACQUISITION/COLLECTION/STORAGE AND TRANSMISSION HARDWARE REQUIREMENTS}

The data acquisition system will be comprised of two general components: The first will be the hardware mounted on the decontamination unit that will record, store, and transmit the data to a remote location; the second will be the hardware associated with the remote monitoring station.

As stated previously, the components selected must be commercially available and their vendors receptive to integration processes. The costs involved must not be prohibitive for the integration process. The final system must be capable of storing the results of a final radiation scan (prior to the final scan from the Health Physics crew), then subsequently transmitting them to a remote monitoring station, which itself will be capable of storing all scans sent by the decontamination unit (i.e., storing the results on a computer hard drive).

All components must be capable of functioning in a radioactive environment.

If the initial design phase finds that integration is not feasible for these components, then the data acquisition system will be eliminated from the final integrated unit. This decision will be made immediately following performance of Subtask 6.c (Feasibility Study) and will be reported with Task 7: First round design of remote monitoring and data collection station.

The primary technical doubts with regards to the feasibility of a data acquisition system, at this point in the project are the following:

- Is commercially available data acquisition hardware capable of withstanding radioactive environments? 
- Can data be transmitted through structures typically found at DOE facilities (thick concrete walls with radioactive backgrounds, steel structures, and possible lead shielding)

- Is data transmission to a remote location an advantageous thing to do? If the data collection components integrated into the decontamination technology can store the data and later be downloaded to a computer, then what would be the advantage of storing it simultaneously at a remote station? 


\section{APPENDIX B}

\section{PRELIMINARY FEASIBILITY STUDY FOR THE INTEGRATION OF A CHARACTERIZATION TECHNOLOGY WITH A DECONTAMINATION TECHNOLOGY}


The feasibility of integrating a decontamination technology with a characterization technology or with a set of characterization technologies is presented within this document. The general need for such an integration is unmistakable since there currently is no existing technology and the processes of radioactive contamination decontamination and contamination characterization are being performed separately in an iterative fashion. This iterative approach is inefficient in that two sets of workers are being deployed into radioactive areas and they are subsequently being exposed to radiation and the odds of spreading radioactive contamination increase with the entry of each group.

The primary challenges to integrating the two above technologies are the following:

- Size constraints

- Electrical constraints

- Dynamic efficiency of radiation detection instruments

- Fragility of radiation detection instruments

- User friendliness in the operation of decontamination systems, radiation detection systems, and the combination of these two types of systems

- Variety of contaminants present at radioactively contaminated sites.

In order to evaluate the feasibility of such an integration, several tasks were defined in a chronological order to lay a foundation. These tasks are outlined in the project PTP and are generally as follows:

1. Delineation of requirements for the final integrated unit.

2. Evaluate decontamination technologies and selection of a short list of technologies that may be used.

3. Evaluate radiation detection instruments and select a subset that may be used for integration.

4. Selection of one decontamination technology for integration.

5. Selection of components/subsystems for integration.

6. First-round design of the integration.

7. First-round design of data collection, transmission, and storage devices.

8. First-round design of remote monitoring station.

9. First-round integration design.

The feasibility of integrating the previous technologies centered largely on overcoming the primary challenges previously listed. After completing the project tasks, these issues were overcome in the following manners:

- Size constraints: The decontamination technology chosen has a blast path width of approx 14 inches. The decon unit measures roughly three feet in height, four feet in length, and a foot and a half in width. The characterization array design was therefore constrained to the width of the unit, as flat as possible (on the floor) and no more than one foot of length trailing behind the decon unit. The electronics, computer, cal gas bottle, and gamma detectors were 
all chosen so that their size could be incorporated right onto the decon unit without adding an extra unit or too much bulk. The integration design is best depicted in the attached CAD drawing.

- Electrical constraints: The decon unit uses 440 volt power. The detectors are run off a 12 -volt battery. Since the difference in voltages and possibly currents was too drastic, it was decided to use a battery supply to run the detectors and computer. It may be decided at a later date to tap into the decon unit electrical system, but for the initial phase it is not necessary. Another related issue is that of EMI. Based on general experience and engineering knowledge, it is not believed that EMI will be an issue (based on vendor information regarding the sensors, electronics, and decon unit). However, plans are being made to rent a generator to power the decon unit and to perform an EMI sweep.

- Dynamic efficiency of the detectors in motion is one of the key elements of the project. Two things have been pursued to evaluate this. The first was to use available vendor data related to detector efficiencies. The data was generated primarily for beta and alpha types of radiation and does present a significant contribution to proving the feasibility of this type of integration. It is also important to point out that the vendor operates a business that is based on the use of such integrated technologies and therefore has much empirical evidence that these types of integrations do work. The second thing being pursued is a detector efficiency feasibility study using an X-Y positioning table and a frame to hold detectors in place while radioactive sources are moved past them. This test will yield the missing data on gamma detector efficiency as a function of scanning speed and distance from the detector. It will be performed with available radiation detection instruments until the commercial detectors can be received in-house for testing.

- Fragility of radiation detectors: One of the technologies first investigated for the integration project was Phoswich detectors. After considerable investigation, it was found that it was indeed possible and feasible. This conclusion was based on purely theoretical data. The final decision not to incorporate this type of detector was made based on empirical evidence: Discussions with a commercial vendor indicated that a Phoswich design being used by them was theoretically designed correctly but when placed into service, the vibrations caused by moving the detector caused an unacceptable level of interference. Therefore, based on this information which came purely from field tests, the decision was made not to use Phoswich technology. This technology may serve however in different applications where there are no excessive vibrations taking place. All other detectors were evaluated and/or chosen based on their ability to withstand some vibration and survive work environments. The characterization array placement was based primarily on a compromise between being as close as possible to the blast path (of the shot blast) and being far enough away to be protected. The result was a location trailing the shot blaster that will yield near-real-time results and still be protected enough to ensure the detectors do not fail. Additionally, the mechanical housing for the detector will be fitted with skirts and protective rubber grommets so that shot does not make its way to the detector faces and penetrate them.

- Since the resulting technology will be used by non-health physics, it is imperative to achieve an acceptable level of user friendliness. For that reason, a computer interface was chosen that uses a program generated specifically to monitor multiple detectors in a dynamic 
environment. The program is proprietary and was designed to be as user friendly as possible. It operates on a laptop computer (connected to the electronics and in turn to the detectors). Positioning data is also fed into the program so that all system outputs can be monitored on one screen. The data is stored on the laptop computer so that there are no separate data loggers or hardware.

- Variety of radioactive contaminants: Early in the project, a list of radioactive isotopes was compiled to be used as target elements. The list was compiled based on examination of DOE needs statements and information from DOE sites. This list was then used to evaluate the applicability of radiation detectors in question. Once the target list had been generated, it was then necessary to decide which of the isotopes would be retained as target contaminants. The decision was made to eliminate the alpha emitters since the detection efficiency would be very low in dynamic applications ( 2 inches away from the contaminants and .5 inches/sec). This left a subset of contaminants that continue to be used as target contaminants. 
EMPIRICAL FEASIBILITY STUDY OF THE EFFECTS OF HORIZONTAL MOTION ON THE EFFICIENCY OF RADIATION SENSORS 


\section{INTRODUCTION}

This study aimed to verify the integration of radioactivity sensors and a shot-blasting technology. The main focus of the investigation was to determine the efficiency loss in the readings of the radioactivity sensors while in motion. The motion discussed is motion parallel to the target surface, as well as the combined effects of motion and distance perpendicular to the target surface.

The study consisted of using a positioning table to move a small Cesium 137 gamma source. A sensor was placed above the source. The distance above was measured, and the position of the sensor was considered to be the origin of the coordinate system. The source was then moved at a known velocity below the sensor. This was performed at speeds ranging from $0.25 \mathrm{in} / \mathrm{sec}$ to 1 in/se.

In addition, the test was performed at different distances from the origin. These ranged from $1 / 8$ " to 2 ". Also, readings for a stationary source were taken when the source was at coordinates $(0, y)$ for values of $y \in\{0,0.25,0.5,0.75,1, \ldots 3.25\}$. The units are inches. The readings at $(0, y)$ were taken at different heights ( $\mathrm{z}$ values) from the origin.

\section{RESULTS}

It was found that there is a decrease in efficiency, approximately $40 \%$. However, this is not a constant quantity. The effect decreases as the distance perpendicular to the surface increases.

The sensor used did not provide measurements with a large number of significant figures. Therefore, the values obtained can not be treated as a standard for calibration. In addition, radioactivity is a random phenomenon. Consequently, a large number of readings would be necessary in order to perform a full statistical analysis and obtain a calibration factor.

Since the medium was not a complete vacuum, the decrease of readings deviated slightly from the inverse square law. Another interesting phenomenon was encountered: The peak of the readings did not coincide with the crossing of the source over the origin. There seems to be some sort of delay within the circuit that accounts for this observation.

The results are found in an excel database, and the logbook for these experiments is also available.

The setup for the feasibility study is shown below. 


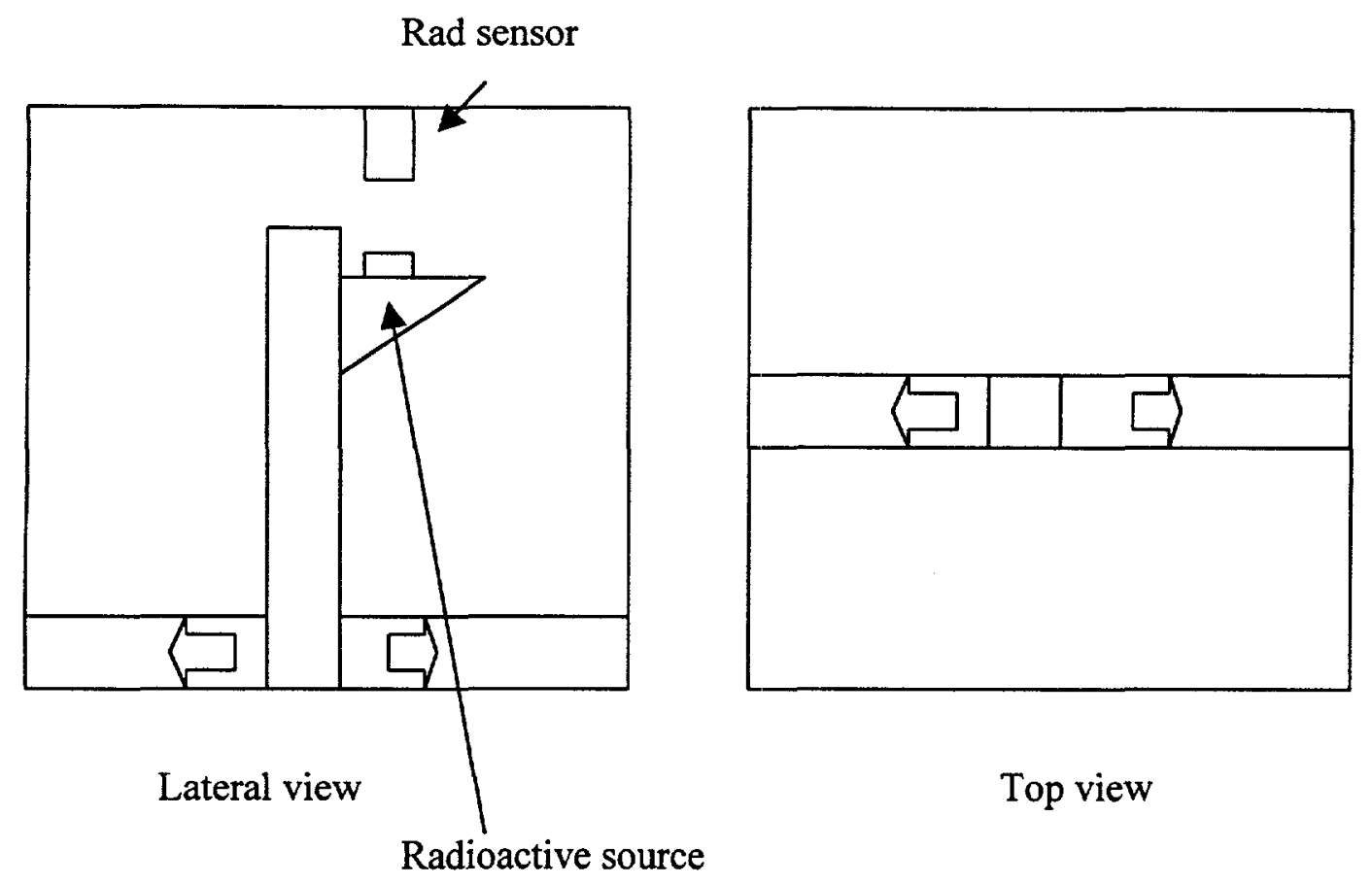

